\title{
Dragon fruit-like biocage as an iron trapping nanoplatform for high efficiency targeted cancer multimodality imaging
}

\author{
Min Yang ${ }^{\text {a, b, } 1}$, Quli Fan ${ }^{\text {b, } 1}$, Ruiping Zhang ${ }^{\text {b, } 1}$, Kai Cheng ${ }^{\text {b }}$, Junjie Yan ${ }^{\text {a }}$, Donghui \\ Pan $^{\mathrm{a}}$, Xiaowei Ma ${ }^{\mathrm{b}}$, Alex Lu ${ }^{\mathrm{b}}$, and Zhen Cheng ${ }^{\mathrm{b}, *}$ \\ ${ }^{\mathrm{a}}$ Key Laboratory of Nuclear Medicine, Ministry of Health, Jiangsu Key Laboratory of Molecular Nuclear \\ Medicine, Jiangsu Institute of Nuclear Medicine, Wuxi 214063, China \\ b Molecular Imaging Program at Stanford (MIPS), Canary Center at Stanford for Cancer Early Detection, \\ Department of Radiology and Bio-X Program, School of Medicine, Stanford University, CA 94305-5484, USA \\ * Corresponding author. 1201 Welch Road, Lucas P095, Stanford, CA 94305-5484, USA \\ E-mail addresses: zcheng@ stanford.edu \\ ${ }^{1}$ These authors contributed equally to this work. \\ Keywords: Apoferritin, Melanin, PET, MRI, PAI
}

\begin{abstract}
Natural biopolymer based multifunctional nanomaterials are perfect candidates for multimodality imaging and therapeutic applications. Conventional methods of building multimodal imaging probe require either cross-linking manners to increase its in vivo stability or attach a target module to realize targeted imaging. In this study, the intrinsic photoacoustic signals and the native strong chelating properties with metal ions of melanin nanoparticle (MNP), and transferrin receptor 1 (TfR1) targeting ability of apoferritin (APF) was employed to construct an
\end{abstract} efficient nanoplatform (AMF) without tedious assembling process. Smart APF shell significantly increased metal ions loading (molar ratio of $1: 800, \mathrm{APF} / \mathrm{Fe}^{3+}$ ) and therefore improved magnetic resonance imaging (MRI) sensitivity. Moreover, synergistic use of $\mathrm{Fe}^{3+}$ and APF contributed to high photoacounstic imaging (PAI) sensitivity. AMF showed excellent bio-stability and presented good in vivo multimodality imaging (PET/MRI/PAI) properties (good tumor uptake, high specificity and high tumor contrast) in HT29 tumor because of its targeting property combined with the enhanced permeability and retention (EPR) effect, making it promising in theranostics 
and translational nanomedicine.

\section{Introduction}

Non-invasive imaging techniques have attracted much attention due to their considerable roles in tumor diagnosis, prognosis and personalized treatment guidance [1-3]. Multimodality imaging provides comprehensive information and accomplishes synergistic advantages over any single modality alone [4-6]. The advance on nanomaterials research has offered numerous opportunity for designing multifunctional nanoprobes with unique chemical and physical properties [7, 8]. Nanomaterials with various building blocks have been explored for multimodal imaging, however, it is usually contradictory to simultaneously satisfy expected demands and avoid intrinsic limitations. For instance, polymeric micelles are with easy synthesis and modification, but they are not suitable for loading of hydrophilic payloads [9-11]. Inorganic nanoparticles possess good biocompatibility, controllable size and shape, but accompanying poor in vivo stability [12-15]. Particularly, there is a growing need to accurately image biological targets (e.g. receptors over-expressed in tumor), which not only increases the cost of imaging probes, but also makes the preparation process troublesome. To address those issues, biological nanoparticles are perfect candidates because of their biodegradability and homogeneity. But their handling and further modification are very difficult [16-18]. Therefore, facile synthesis and construction of efficient multimodal imaging platforms is very significant.

As indispensable elements for living organisms, metal ions are also appropriate reporters for bioimaging because of their attractive physical characteristics such as magnetic, optical and radioactive properties $[19,20]$. However, free metal ions show weak in vivo stability and cause 
severe toxixicity, therefore metal ions are usually bound with or encapsulated into a carrier. Melanin nanoparticle (MNP) is such a carrier that not only shows intrinsic chelating ability with metal ions $\left({ }^{64} \mathrm{Cu}^{2+}, \mathrm{Fe}^{3+}\right)$ for positron emission tomography (PET) and magentic resonance imaging (MRI) with a high loading capacity, but also possesses native optical properties for photoacoustic imaging (PAI) [21]. However, MNP complexed with metal ions could precipitate in aqueous solutions, and PEGylation was utilized to solve this problem and increase the water solubility of MNP. Moreover, in order to make naoparticles access to targeted tissues, apart from enhanced permeability and retention (EPR) effect, attaching a targeting moiety such as folic acid or RGD peptide is usually necessary [22-25], which makes the preparation process troublesome and also could result in heteogenous products .

Ferritin is a natural iron storage protein. It shows cage-like structure with small size (around $10 \mathrm{~nm})$, large surface for modification, multi-channel $(<1 \mathrm{~nm})$ connecting the exterior with interior, dissociation-reassemble characters upon $\mathrm{pH}$ changes, making it a widely used nanoplatform for metal capture, drug delivery, bioimaging techniques and phototheramal therapy [18, 26-28]. More importantly, it possesses targeting ability to transferrin receptor 1 (TfR1) which is overexpressed in numerous type of cancer cells [29]. Ferritin that is not combined with iron is called apoferritin, and it is extensively utilized for cargo loading. These features suggest ferritin a smart platform with intrinsic targeting ability.

Herein, we report the integrated endogenous nanomaterials for enhanced targeted multimodality imaging through ingeniously space matching, i.e., embedding ultrasmall MNPs into the cavity of apoferritin (APF). APF played synergistic roles of guaranteeing targeting and stability of the nanoplatform. First, we prepared ultra-small and water-soluble MNPs and then 
encapsulated one MNP together with high concentration of ferric irons into the cavity of APF to prepare nanomaterials AMF with core-shell structures. ${ }^{64} \mathrm{Cu}^{2+}$ and $\mathrm{Fe}^{3+}$ can be easily loaded into AMF nanoparticles cavities without adding additional chelating agents. More importantly, AMF can achieve TfR1 targeted cancer trimodal (PET/MRI/PAI) imaging (Fig. 1). To the best of our knowledge, AMF represents the first organic nanoplatform integrating both melanin and apoferritin with high and stable metal ions load for targeted cancer molecular imaging.

\section{Results and discussion}

\subsection{Preparation and characterization of $A M F$}

The inside cavity diameter of APF is $\sim 8 \mathrm{~nm}$, thus, ultrasmall MNPs below $8 \mathrm{~nm}$ were synthesized from commercial melanin granules (Supporting Information) according to previous report [21]. The average sizes of MNPs were $4.0 \mathrm{~nm}$ measured by transmission electron microscopy (TEM) (Fig. 2B). Hydrodynamic diameters of MNPs were $~ 5.6 \mathrm{~nm}$ determined by dynamic light scattering (DLS, Fig. 2E), and zeta potential of MNP was -31.8 mV (Table S1). The molecular weight of MNP was determined to be around $61 \mathrm{kDa}$ by matrix-assisted laser desorption/ionization time of flight mass spectrometry (MALDI-TOF-MS) (Fig. S2).

APF cage shows reversible $\mathrm{pH}$-responsiveness, it disassembles into discrete subunits in strong acidic environment ( $\mathrm{pH} 2.0$ or 3.5), and re-assembles to a cage-like structure under basic conditions ( $\mathrm{pH}$ 8) [30-32]. This smart architecture renders it convenient to encapsulate MNP and $\mathrm{Fe}^{3+}$ within the cavity of APF to prepare AMF (Fig. 1). Usually, the loading yields of metal ions into apoferritin are very low; for example, it was reported that about 8-10 Gd-HPDO3A molecules and one molecule of [DFOFe] complex were trapped within an APF molecule [33, 34]. Since MR signal is positively associated with $\mathrm{Fe}^{3+}$, higher ferric ion capture is preferred. APF:Melanin:Fe ${ }^{3+}$ 
at different ratios were used to prepare AMF, and the optimal conditions were determined as 1:1:1000 (Supporting Information). The loading yield of $\mathrm{Fe}^{3+}$ to $\mathrm{AMF}$ was $71.36 \pm 2.56 \%$, achieving a molar ratio of 1:1:800 APF:MNP:Fe ${ }^{3+}$. This was a very high loading. Previously, we found MNP quickly precipitated after adding $\mathrm{Fe}^{3+}$. Even after PEGylation, the maximum ratio of $\mathrm{Fe}^{3+}$ to MNP-PEG was only around 90:1 [21]. In this study, the solution containing APF remained a clear brown solution at room temperature for at least 1 month (Fig. S3), whereas black precipitate was immediately observed in APF-free condition. On the other hand, in a MNP-free solution, $\mathrm{Fe}^{3+}$ was stably encapsulated in acid condition, but after transfer to neutral or basic condition, $\mathrm{Fe}^{3+}$ could leak freely from APF through channels between the exterior and interior cavities, resulting in red-brown precipitates. Therefore, in this circumstance, the final $\mathrm{Fe}^{3+}$ loading yield within APF alone was only 4\%. In contrast, using the APF-MNP dual platform, $\mathrm{Fe}^{3+}$ ion loading increased more than 10 times and MNP inside APF dramatically enhanced the stability of $\mathrm{Fe}^{3+}$ loading.

The nanomaterials prepared in aqueous solution were examined by TEM and DLS. APF showed in Fig. 2A was stained with uranyl acetate, which cannot penetrate the protein shell and therefore cannot stain the cavity, revealing APF as lighter round patches, which was consistent with previous results.[30-32] The diameter was $\sim 12.1 \mathrm{~nm}$ measured by TEM, the hydrodynamic diameter and zeta potential were $\sim 13.6 \mathrm{~nm}$ and $-17.2 \mathrm{mV}$ respectively measured by DLS (Table S1). Fig. 2D showed that the AMF nanoparticle had the similar core-shell structure as APF, the diameter increased to $16.4 \mathrm{~nm}$ (Fig. 2E) and zeta potential of AMF was $-18.4 \mathrm{mV}$, indicating both MNP and $\mathrm{Fe}^{3+}$ were encapsulated inside the protein cavity. Fig. 2C showed a non-stained sample of AMF, discrete electron-dense sphere was clearly observed and the diameter was $\sim 6 \mathrm{~nm}$, which 
was a little larger than MNP (Fig. 2B), this suggested $\mathrm{Fe}^{3+}$ was chelated by MNP inside the cavity of AMF. This was consistent with our previous work that the diameter of PEG-MNP increased from $7.0 \mathrm{~nm}$ to $8.9 \mathrm{~nm}$ after $\mathrm{Fe}^{3+}$ loading [21]. All these confirmed that one MNP and many $\mathrm{Fe}^{3+}$ ions were successfully loaded into the cage of APF.

\subsection{Stability of $A M F$}

Next, we investigated the stability of AMF. The synthesized AMF brown solution was subjected to dialysis in either phosphate-buffered saline (PBS, $1 \times, \mathrm{pH} 7.4)$ or acetate buffer $(\mathrm{pH}=5)$. At $\mathrm{pH}$, precipitation was observed within the dialysis bag (molecular weight cut-offs: 3 $\mathrm{k}$ and $100 \mathrm{k}$ were both used in $\mathrm{pH} 7.4$ and $\mathrm{pH}$ 5) after $1 \mathrm{~h}$, while no color changed at $\mathrm{pH}$ 7. The dialysis process was followed by UV-VIS spectroscopy and ICP-MS analysis. No $\mathrm{Fe}^{3+}$ or MNP release were detected within the dialysis solution out of bag within $120 \mathrm{~h}$ at $37{ }^{\circ} \mathrm{C}$ (Fig. S4), which clearly indicated that the Fe-MNP complex remained trapped inside the APF at pH 7 for long periods. The diameter of Fe-MNP complex was about $6 \mathrm{~nm}$, inhibiting its leakage from the APF channels $(<1 \mathrm{~nm})$. At $\mathrm{pH} 5$, the protein cage disassembled, Fe-MNP was released from APF cage, however, the precipitated Fe-MNP complex was not separated, neither free $\mathrm{Fe}^{3+}$ nor melanin were detected out of the dialysis bag.

\subsection{Stability of ${ }^{64} \mathrm{Cu}-\mathrm{AMF}$}

After stable and water-soluble AMF was prepared, ${ }^{64} \mathrm{Cu}^{2+}$ was selected to label AMF for PET imaging due to its moderate half-life $(12.7 \mathrm{~h})$. Traditionally, the labeling of ${ }^{64} \mathrm{Cu}^{2+}$ is performed by preconjugating chelators such as 1,4,7,10-tetraazacyclododecane-1,4,7,10-tetraacetic acid (DOTA) with the platform in a multistep process [35-37]. In this study, ${ }^{64} \mathrm{Cu}^{2+}$ was loaded directly into AMF at pH 6 through multichannels of APF without any linker or nanocage disassembly. A dose 
calibrator (Capintec) showed that $80 \%$ of the radionuclide could be loaded into AMF after 30 min incubation at room temperature. This loading yield was higher than the previous report ( $60 \%)$ [18]. To probe the stability of the loaded ${ }^{64} \mathrm{Cu}^{2+},{ }^{64} \mathrm{Cu}^{2+}$-labeled AMF was then subjected to dialysis in either PBS or fetal bovine serum (FBS), and the radioactivity decay was monitored over time. In both media, ${ }^{64} \mathrm{Cu}^{2+}$ was well retained in the nanocages (Fig. S4), with less than $10 \%$ of the radioactivity (decay-corrected) released from the nanocages within the observation period of $24 \mathrm{~h}$.

\subsection{In vitro cell studies}

Cell line HT29 has been reported to overexpress transferrin receptor 1 (TfR1), while HepG2 has low TfR1 expression [38, 39]. Therefore, HT29 and HepG2 cells were further used for cell uptake and blocking studies to investigate the targeting specificity of ${ }^{64} \mathrm{Cu}$-AMF. As shown in Fig. $\mathrm{S} 8$, the uptake of ${ }^{64} \mathrm{Cu}-\mathrm{AMF}$ decreased significantly $(\mathrm{P}<0.05)$ at 1 and $2 \mathrm{~h}$ in HT29 cell lines incubated with the blocking dose of free APF $(1 \mu \mathrm{M})$, and their uptakes were close to those in HepG2 cells. By contrast, the uptake of ${ }^{64} \mathrm{Cu}-\mathrm{AMF}$ in HepG2 cells didn't show obvious alteration with or without APF blocking. These results indicate that APF coated nanoparticles specifically recognize the transferring receptor positive cells. Moreover, competitive binding of ${ }^{64} \mathrm{Cu}-\mathrm{AMF}$ to HT29 cells was determined using APF as competitor (Fig. S9). The apparent eqilibrium dissociation constant was calculated and $\mathrm{Kd}$ value for ${ }^{64} \mathrm{Cu}-\mathrm{AMF}$ was $20.2 \mathrm{pM}$. Lastly, MTT assay also indicates that AMF has high biocomatability. No observed toxicity to the cells at even $10 \mu \mathrm{M}$ of AMF (Fig. S11).

\subsection{In vivo imaging}


After confirming the structural composition and stability of ${ }^{64} \mathrm{Cu}-\mathrm{AMF}$, in vivo validation experiments were conducted. In brief, the nanoprobe was injected intravenously into tumor-bearing mice and then imaged by PET, MRI and PAI. Human colon cancer HT29 [38] overexpressing TfR1 and liver cancer HepG2 with low TfR1 expression [39] were used to set-up animal models for PET/MRI/PAI imaging.

\subsubsection{PET imaging}

For PET studies, mice $(n=4)$ were injected with about $100 \mu \mathrm{Ci}$ of ${ }^{64} \mathrm{Cu}-\mathrm{AMF}$ via tail vein. Tomographic images were acquired on a Siemens Inveon microPET/CT scanner at various time points. The decay-corrected whole body images were shown in Fig. 3. It can be observed that the signal intensities gradually increased in the HT29 tumor area and reached maximum at $4 \mathrm{~h}$ post-injection (p.i.), followed by very slow signal intensity decrease (Fig. 3A). Quantification analysis of three-dimensional regions of interest (ROIs) over the images showed that the probe's biodistribution pattern was consistent with that previous reported [18, 21], and ${ }^{64} \mathrm{Cu}-\mathrm{AMF}$ showed high accumulation in liver, which indicated that AMF was cleared mainly through hepatobiliary system. Moreover, ${ }^{64} \mathrm{Cu}-\mathrm{AMF}$ displayed higher tumor uptakes in comparison to RGD-modified melanin, and the tumor uptakes were $4.82 \pm 0.59,6.14 \pm 0.77,7.34 \pm 0.93,7.26 \pm 1.32$, and $6.74 \pm$ $0.51 \% \mathrm{ID} / \mathrm{g}$ at 1, 2, 4, 18 and 26 h p.i., respectively (Fig. S10). Such high tumor accumulations were possibly caused by synergistic apoferritin-TfR1 specific interaction (Fig. S8) and EPR effect. To verify this, HepG2 liver cancer model was used as a negative control $(n=4)$. Significant lower tumor uptakes were observed at all time points, which were $2.95 \pm 0.40,3.55 \pm 0.58,4.33 \pm 1.16$, $4.61 \pm 1.58$ and $4.77 \pm 0.47 \% \mathrm{ID} / \mathrm{g}$ at $1,2,4,18$ and $26 \mathrm{~h}$ p.i., respectively. This observation corroborated our hypothesis that specific targeting was involved in the accumulation and retention 
of AMF in the TfR1 positive tumor. The uptakes of ${ }^{64} \mathrm{Cu}-\mathrm{AMF}$ in most normal tissues were very low after $24 \mathrm{~h}$, with persistent high uptake only in the liver (the site of transferrin production and metabolism). The liver uptakes of HT29 and HepG2 models were comparable $(\mathrm{P}>0.05)$, reaching a peak of 1-2 h p.i before decreasing. Even after 48 h p.i., around 15 ID\%/g liver uptakes were observed, confirming liver as the main metabolism and retaining organ for ${ }^{64} \mathrm{Cu}$ and nanoparticles [40-42].

\subsubsection{MRI imaging}

In MRI studies, relaxation times of the phantoms were first measured. By determining the variation in relaxation times of different concentrations of AMF, the relaxation rates (reciprocal values of relaxation times) were plotted against the amount of $\mathrm{Fe}^{3+}\left(1 / \mathrm{T}\right.$ vs. $\left.\left[\mathrm{Fe}^{3+}\right]\right)$. Relaxivity values ( $\mathrm{r} 1$; the slopes of each plot, $\mathrm{mM}^{-1} \mathrm{~s}^{-1}$ ) were determined to be 2.54 by using $\mathrm{FeCl}_{3}$ as control and saturation test software in the 1.5T Bruker micro-MR system (Fig. S5). This value is over 2 fold compared to the $\mathrm{r} 1$ value of Fe-RGD-PEG-MNP $\left(1.2 \mathrm{mM}^{-1} \mathrm{~s}^{-1}\right)$ [21], this high loading efficiency of $\mathrm{Fe}^{3+}$ in AMF can largely improved MRI sensitivity. Tumor bearing mice $(\mathrm{n}=4)$ were then administered 10 nmol AMF through the tail vein. Significantly enhanced signals in HT29 tumor were observed with times ranging from 1 to 4 h. (Fig. 4). Ratios of HT29 tumor uptake after injection vs. pre-scan levels $\left(\mathrm{T} / \mathrm{T}_{0}\right)$ were $1.58,1.73$ and 2.02 at 1,2 and $4 \mathrm{~h}$ p.i., respectively. However, HepG2 tumor mice showed lower signals with quick clearance of nanoprobes, the corresponding $\mathrm{T} / \mathrm{T}_{0}$ ratios were $1.21,1.44$ and 1.03 at 1,2 and $4 \mathrm{~h}$ p.i., respectively. These results demonstrated that AMF can be successfully used for targeted MRI of tumor, and they also confirmed that AMF helped the uptake in TfR1 positive tumors.

\subsubsection{PAI imaging}


To study the PAI applications of AMF, we first tested the photoacoustic intensities of a tube phantom. Different concentrations of MNP, Fe-PEG-MNP, AMF, and AMF without Fe (APF+MNP) were loaded into separate tubes (Supporting Information). The AMF signal was about 2 times higher than the other 3 nanoparticles $(\mathrm{p}<0.01)$, reaching maximum emission strength at $500 \mu \mathrm{g} / \mathrm{mL}$ (based on MNP concentration) (Fig. S6). These data suggested that the strong signal intensity of AMF stemmed from the combination of APF and Fe, and AMF has higher PAI sensitivity than MNP, Fe-PEG-MNP, and AMF without Fe. Furthermore, in vivo imaging studies were performed in HT29 mice models $(n=4)$. After injection of 20 nmol AMF, PAI contrast increased with time in HT29 tumor (Fig. 5). The tumor uptake reached $1.03 \pm 0.02$, $7.31 \pm 2.33$ and $24.15 \pm 2.86$ A.U. at 0,2 and 4 h p.i., respectively. In contrast, no obvious PAI contrast was observed in the negative HepG2 tumor bearing mice (Fig. S12). These data clearly indicated that AMF was an excellent nanoprobe for PAI in addition to its capability for PET and MRI.

\section{Conclusion}

In summary, we report $\mathrm{AMF}$ as a nanoplatform for high efficiency targeted cancer multimodality imaging. APF is a focusing heat point because of its pH-dependant disassembly-assembly behavior and TfR1 targeting ability can behave as an efficient platform to simplify the combining of loading different imaging moieties and control the size of nanomaterial. Combining APF and MNP can increase the metal ions loading and improve the stability of the nanoplatform. At last but not least, AMF presented good in vivo tumor imaging properties. The nanoplatform is safe with complete utilization of natural materials, which is important for future translation into clinic use. The technique developed in this work can be potentially extended to 
load other types of functionalities such as imaging agents (Gd, ${ }^{89} \mathrm{Zr}, \mathrm{Mn}$ and etc.), therapeutic radionuclides $\left({ }^{90} \mathrm{Y},{ }^{177} \mathrm{Lu}\right.$ and etc.) and targeting motifs (peptides, affibody, antibody and etc.).

\section{Experimental section}

\subsection{Chemicals and reagents}

Apoferritin (APF) from equine spleen (A3641, $50 \mathrm{mg} / \mathrm{mL})$ and melanin (M8631) were both obtained from Sigma-Aldrich. PD-10 column (17-0851-01), NAP-5 columns were purchased from GE Health, and Slide-A-Lyzer® Dialysis Cassettes, 100-500 $\mu 1,3500$ MWCO (66335) from Thermo Fisher Scientific.

\subsection{Preparation of small melanin nanoparticles (MNPs)}

Ultra small and water soluble melanin nanoparticles were prepared according to the previous report [21]. Melanin was first dissolved in $1 \mathrm{M} \mathrm{NaOH}$ aqueous solution before adjusting $\mathrm{pH}$ to 7 using aqueous $1 \mathrm{M} \mathrm{HCl}$ solution under strong sonication. The bright black melanin aqueous solution was further purified and lyophilized to obtain black MNPs in solid state. The diameter was measured in water by Dynamic Light Scattering (DLS) using the ZetaSizer Nano-ZS (Malvern), and TEM images were taken using the JEOL JEM-2010 transmission electron microscope. Mass of MNPs was measured using Matrix-Assisted Laser Desorption/Ionization Time of Flight Mass Spectrometry (MALDI-TOF-MS) in Stanford University.

\subsection{Preparation of melanin-Fe-loaded apoferritin nanoparticles (AMF)}

Different ratios of APF, MNP and $\mathrm{Fe}^{3+}$ were tested to identify the optimal conditions (Fig. S3) before selecting 1:1:1000. APF was first diluted with water (90 nmol in $4 \mathrm{~mL}$ water). The solution $\mathrm{pH}$ was then adjusted to 2 with $1 \mathrm{M} \mathrm{HCl}$ while gently shaking at room temperature. MNP (90 nmol in $5 \mathrm{ml}$ water $)$ and $\mathrm{FeCl}_{3}(90 \mu \mathrm{mol}$ in $135 \mu \mathrm{L}$ acidic water $)$ were added into the acid solution. The 


\subsection{Preparation of ${ }^{64} \mathrm{Cu}-\mathrm{AMF}$}

Contrary to the literature $[18],{ }^{64} \mathrm{Cu}$ radiolabeled AMF without disrupting the cage at $\mathrm{pH}=2$. ${ }^{64} \mathrm{CuCl}_{2}(1 \mathrm{mCi})$ was added into AMF solution directly. The mixture was incubated at temperature for 30 minutes. The products were then purified by PD-10 column to remove free ${ }^{64} \mathrm{Cu}^{2+}$. The fractions eluted from PD-10 were measured for radioactivity and the most concentrated fractions were used for animal experiments. For a stability test, purified ${ }^{64} \mathrm{Cu}-\mathrm{AMF}(300 \mu \mathrm{l}, \sim 100 \mu \mathrm{Ci}$, respectively) were added to a $500 \mu \mathrm{L}$ mini-dialysis unit. The samples were then dialyzed against $10 \mathrm{~mL}$ PBS buffer or FBS. The radioactivity remaining in the dialysis units and those leaked into the outer solution were both measured by a radioisotope calibrator.

\subsection{PET imaging}

The details of small-animal PET imaging and region-of-interest (ROI) analysis have been reported previously [43]. In brief, each mouse was injected with about $100 \mu \mathrm{Ci}$ of ${ }^{64} \mathrm{Cu}-\mathrm{AMF}$ via tail vein. Mice bearing tumors $(\mathrm{n}=4)$ underwent microPET/CT (Seimens Inveon) scans at various time points after the injection. For each microPET/CT scan, 3-dimensional ROIs were drawn over the tumor or other normal organs on decay-corrected whole body coronal images. The $\% \mathrm{ID} / \mathrm{g}$ was then derived from the readings by IAW software.

\subsection{MR imaging}

Phantom Imaging: AMF was prepared in Eppendorf tubes at varying concentrations for 
imaging. The $\mathrm{T}_{1}$ relaxation times were measured on a $1.5 \mathrm{~T}$ Bruke $\mathrm{MRI}$ scanner. $\mathrm{FeCl}_{3}$ was measured in the same manner as control. The parameters were as follows: TR/TE=50, 100, 200, 500, 750, 1000, 1500, 2000/8.8 ms; SI: $3.0 \mathrm{~mm}$; FOV: $6.0 \mathrm{~cm}$; MTX: 256/128). The signal intensities for each of the ROIs on the $\mathrm{T}_{1}$ map were measured for each concentration. $\mathrm{T}_{1}$ saturation method within the machine was selected to calculate $T_{1}$ values. Specific relaxivities (r1) of AMF or $\mathrm{FeCl}_{3}$ were calculated from the plots of $\mathrm{T}_{1}^{-1}$ versus concentration of $\mathrm{Fe}$ in $\mathrm{AMF}$ or $\mathrm{FeCl}_{3}$.

Small animal MR Imaging: The animals $(n=4)$ were anesthetized and set into the $1.5 \mathrm{~T}$ micro-MR-compatible cradle. During MRI, the animal was anesthetized with inhalation of $2 \%$ isoflurane with oxygen-enriched air through a facemask. Temperature was maintained at $36 \pm$ $1{ }^{\circ} \mathrm{C}$ using a water bath underneath the cradle and electrocardiogram (ECG) was used to detect the heart rate. The prepared AMF (10 nmol/200 $\mu \mathrm{L}$ per mouse) were intravenously administered. To investigate the time course distributions of AMF in the mouse body, MRI was performed before and 1, 2 and $4 \mathrm{~h}$ after the administrations. High-resolution AMF contrast-enhanced multislice MR images were obtained from each tumor by using $\mathrm{T}_{1}$-flash MRI sequence: repetition time $(\mathrm{TR}) /$ echo time $(\mathrm{TE})=300 / 6.1 \mathrm{~ms}$; number of experiment $(\mathrm{NEX})=16$; Matrix: $256 \times 256$; slice thickness: $1.25 \mathrm{~cm}$; FOV:3.0 cm; and 15 slices. All images were analyzed using ImageJ software. ROIs were drawn over the tumor and the MR signal was quantified as SItumor, where SItumor is the mean signal intensity of the tumor accumulation. The SI ratio at time interval after AMF injected was calculated as $\mathrm{T} / \mathrm{T}_{0}$.

\subsection{Photoacoustic imaging}

PA/Ultrasound (US) coregistered images were acquired with a LAZR commercial instrument (VevoLAZR; VisualSonics) equipped with a LZ-250 linear array transducer and a tunable 
Nd:YAG laser system (680-970 nm) [44]. Polyethylene (PE) tubes with $1 \sim 2 \mathrm{~cm}$ lengths and 0.7 $\mathrm{mm}$ inner diameters were heat-sealed at one end, then filled with water, $\mathrm{FeCl}_{3}, \mathrm{MNP}$, Fe-PEG-MNP, Apoferritin-MNP-Fe (AMF), AMF without Fe (Apoferritin-MNP), and finally heat-sealed on the opposing side. According to previous report [21], we selected the laser excitation wavelength as $680 \mathrm{~nm}$. Mice bearing HT29 tumors $(\mathrm{n}=4)$ were imaged at $680 \mathrm{~nm}$ before and 2 and $4 \mathrm{~h}$ after the intravenous injection of $20 \mathrm{nmol}$ AMF. Images were exported as 3-dimensional volume tiff files that were stacked together using ImageJ. Normalization and quantification of the tumor images were also done in ImageJ. The PA signals reported were the average pixel intensities from within the ROI.

\section{Acknowledgement}

This work was supported in part by the Office of Science (BER), U.S. Department of Energy (DE-SC0008397), NCI of Cancer Nanotechnology Excellence Grant (CCNE-TR U54 CA119367, CA151459), National Natural Science Foundation of China (NSFC) (81171399, 51473071, 81472749, 81401450 and 21401084), National Significant New Drugs Creation Program (2012ZX09505-001-001), Jiangsu Province Foundation (BE2012622, BL2012031, BE2014609 and BM2012066), Outstanding Professional Fund of Health Ministry in Jiangsu Province (RC2011095, Q201529), CSC foundation (2011832173).

\section{Appendix A. Supplementary data}

Supplementary data related to this article can be found at http:// dx.doi.org/10.1016/

\section{References}

[1] Caobelli F, Bengel FM. In vivo evaluation of atherosclerotic plaques and culprit lesions using noninvasive techniques. Nature Reviews Cardiology. 2015;12:79-U31. 
[2] Ghosh D, Bagley AF, Na YJ, Birrer MJ, Bhatia SN, Belcher AM. Deep, noninvasive imaging and surgical guidance of submillimeter tumors using targeted M13-stabilized single-walled carbon nanotubes. Proceedings of the National Academy of Sciences of the United States of America. 2014;111:13948-53.

[3] Hernandez FJ, Huang LY, Olson ME, Powers KM, Hernandez LI, Meyerholz DK, et al. Noninvasive imaging of Staphylococcus aureus infections with a nuclease-activated probe. Nature Medicine. 2014;20:301-6.

[4] Louie AY. Multimodality Imaging Probes: Design and Challenges. Chemical Reviews. 2010;110:3146-95.

[5] Zhang YM, Jeon M, Rich LJ, Hong H, Geng JM, Zhang Y, et al. Non-invasive multimodal functional imaging of the intestine with frozen micellar naphthalocyanines. Nat Nanotechnol. 2014;9:631-8.

[6] Thorek DLJ, Ulmert D, Diop NFM, Lupu ME, Doran MG, Huang RM, et al. Non-invasive mapping of deep-tissue lymph nodes in live animals using a multimodal PET/MRI nanoparticle. Nature Communications. 2014;5.

[7] Cai WB, Chen XY. Nanoplatforms for targeted molecular imaging in living subjects. Small. $2007 ; 3: 1840-54$

[8] Park K, Lee S, Kang E, Kim K, Choi K, Kwon IC. New Generation of Multifunctional Nanoparticles for Cancer Imaging and Therapy. Advanced Functional Materials. 2009; 19:1553-66.

[9] Savic R, Luo LB, Eisenberg A, Maysinger D. Micellar nanocontainers distribute to defined cytoplasmic organelles. Science. 2003;300:615-8. 
[10] Min KH, Kim JH, Bae SM, Shin H, Kim MS, Park S, et al. Tumoral acidic pH-responsive MPEG-poly(beta-amino ester) polymeric micelles for cancer targeting therapy. Journal of Controlled Release. 2010;144:259-66.

[11] Koo H, Lee H, Lee S, Min KH, Kim MS, Lee DS, et al. In vivo tumor diagnosis and photodynamic therapy via tumoral $\mathrm{pH}$-responsive polymeric micelles. Chemical Communications. 2010;46:5668-70.

[12] Kim J, Piao Y, Hyeon T. Multifunctional nanostructured materials for multimodal imaging, and simultaneous imaging and therapy. Chemical Society Reviews. 2009;38:372-90.

[13] Lee S, Cha EJ, Park K, Lee SY, Hong JK, Sun IC, et al. A near-infrared-fluorescence-quenched gold-nanoparticle imaging probe for in vivo drug screening and protease activity determination. Angewandte Chemie-International Edition. 2008;47:2804-7.

[14] Qian XM, Nie SM. Single-molecule and single-nanoparticle SERS: from fundamental mechanisms to biomedical applications. Chemical Society Reviews. 2008;37:912-20.

[15] Sun IC, Eun DK, Koo H, Ko CY, Kim HS, Yi DK, et al. Tumor-Targeting Gold Particles for Dual Computed Tomography/Optical Cancer Imaging. Angewandte Chemie-International Edition. 2011;50:9348-51.

[16] MaHam A, Tang ZW, Wu H, Wang J, Lin YH. Protein-Based Nanomedicine Platforms for Drug Delivery. Small. 2009;5:1706-21.

[17] Neumann E, Frei E, Funk D, Becker MD, Schrenk HH, Muller-Ladner U, et al. Native albumin for targeted drug delivery. Expert Opinion on Drug Delivery. 2010;7:915-25. 
[18] Lin X, Xie J, Niu G, Zhang F, Gao HK, Yang M, et al. Chimeric Ferritin Nanocages for Multiple Function Loading and Multimodal Imaging. Nano Letters. 2011;11:814-9.

[19] Hong-Hermesdorf A, Miethke M, Gallaher SD, Kropat J, Dodani SC, Chan J, et al. Subcellular metal imaging identifies dynamic sites of $\mathrm{Cu}$ accumulation in Chlamydomonas. Nature Chemical Biology. 2014;10:1034-42.

[20] Topete A, Alatorre-Meda M, Iglesias P, Villar-Alvarez EM, Barbosa S, Costoya JA, et al. Fluorescent Drug-Loaded, Polymeric-Based, Branched Gold Nanoshells for Localized Multimodal Therapy and Imaging of Tumoral Cells. Acs Nano. 2014;8:2725-38.

[21] Fan QL, Cheng K, Hu X, Ma XW, Zhang RP, Yang M, et al. Transferring Biomarker into Molecular Probe: Melanin Nanoparticle as a Naturally Active Platform for Multimodality Imaging. Journal of the American Chemical Society. 2014;136:15185-94.

[22] Lee ES, Na K, Bae YH. Polymeric micelle for tumor $\mathrm{pH}$ and folate-mediated targeting. Journal of Controlled Release. 2003;91:103-13.

[23] Xia W, Low PS. Folate-Targeted Therapies for Cancer. Journal of Medicinal Chemistry. 2010;53:6811-24.

[24] Ruoslahti E, Bhatia SN, Sailor MJ. Targeting of drugs and nanoparticles to tumors. J Cell Biol. 2010;188:759-68.

[25] Lee HY, Li Z, Chen K, Hsu AR, Xu CJ, Xie J, et al. PET/MRI dual-modality tumor imaging using arginine-glycine-aspartic (RGD) - Conjugated radiolabeled iron oxide nanoparticles. J Nucl Med. 2008;49:1371-9.

[26] Zhen ZP, Tang W, Guo CL, Chen HM, Lin X, Liu G, et al. Ferritin Nanocages To Encapsulate and Deliver Photosensitizers for Efficient Photodynamic Therapy against 
Cancer. Acs Nano. 2013;7:6988-96.

[27] Aime S, Frullano L, Crich SG. Compartmentalization of a gadolinium complex in the apoferritin cavity: A route to obtain high relaxivity contrast agents for magnetic resonance imaging. Angewandte Chemie-International Edition. 2002;41:1017-19.

[28] Huang P, Rong PF, Jin A, Yan XF, Zhang MG, Lin J, et al. Dye-Loaded Ferritin Nanocages for Multimodal Imaging and Photothermal Therapy. Adv Mater. 2014;26:6401-8.

[29] Li L, Fang CJ, Ryan JC, Niemi EC, Lebron JA, Bjorkman PJ, et al. Binding and uptake of $\mathrm{H}$-ferritin are mediated by human transferrin receptor-1. Proceedings of the National Academy of Sciences of the United States of America. 2010;107:3505-10.

[30] Yan F, Zhang Y, Yuan HK, Gregas MK, Vo-Dinh T. Apoferritin protein cages: a novel drug nanocarrier for photodynamic therapy. Chemical Communications. 2008:4579-81.

[31] Ji TJ, Zhao Y, Wang J, Zheng X, Tian YH, Zhao YL, et al. Tumor Fibroblast Specific Activation of a Hybrid Ferritin Nanocage-Based Optical Probe for Tumor Microenvironment Imaging. Small. 2013;9:2427-31.

[32] Lin X, Xie J, Zhu L, Lee S, Niu G, Ma Y, et al. Hybrid Ferritin Nanoparticles as Activatable Probes for Tumor Imaging. Angewandte Chemie-International Edition. 2011;50:1569-72.

[33] Crich SG, Bussolati B, Tei L, Grange C, Esposito G, Lanzardo S, et al. Magnetic resonance visualization of tumor angiogenesis by targeting neural cell adhesion molecules with the highly sensitive gadolinium-loaded apoferritin probe. Cancer Research. 2006;66:9196-201.

[34] Dominguez-Vera JM. Iron(III) complexation of Desferrioxamine B encapsulated in apoferritin. J Inorg Biochem. 2004;98:469-72. 
[35] Jarrett BR, Correa C, Ma KL, Louie AY. In Vivo Mapping of Vascular Inflammation Using Multimodal Imaging. Plos One. 2010;5.

[36] Mitchell N, Kalber TL, Cooper MS, Sunassee K, Chalker SL, Shaw KP, et al. Incorporation of paramagnetic, fluorescent and PET/SPECT contrast agents into liposomes for multimodal imaging. Biomaterials. 2013;34:1179-92.

[37] Jarrett BR, Gustafsson B, Kukis DL, Louie AY. Synthesis of Cu-64-labeled magnetic nanoparticles for multimodal imaging. Bioconjugate Chemistry. 2008;19:1496-504.

[38] Fan KL, Cao CQ, Pan YX, Lu D, Yang DL, Feng J, et al. Magnetoferritin nanoparticles for targeting and visualizing tumour tissues. Nat Nanotechnol. 2012;7:459-64.

[39] Sun CJ, Yang H, Yuan Y, Tian X, Wang LM, Guo Y, et al. Controlling Assembly of Paired Gold Clusters within Apoferritin Nanoreactor for in Vivo Kidney Targeting and Biomedical Imaging. Journal of the American Chemical Society. 2011;133:8617-24.

[40] Kaplan JH, Lutsenko S. Copper Transport in Mammalian Cells: Special Care for a Metal with Special Needs. J Biol Chem. 2009;284:25461-5.

[41] Longmire M, Choyke PL, Kobayashi H. Clearance properties of nano-sized particles and molecules as imaging agents: considerations and caveats. Nanomedicine-Uk. 2008;3:703-17.

[42] Choi HS, Liu W, Misra P, Tanaka E, Zimmer JP, Ipe BI, et al. Renal clearance of quantum dots. Nat Biotechnol. 2007;25:1165-70.

[43] Habte F, Ren G, Doyle TC, Liu HG, Cheng Z, Paik DS. Impact of a Multiple Mice Holder on Quantitation of High-Throughput MicroPET Imaging With and Without $\mathrm{Ct}$ Attenuation Correction. Mol Imaging Biol. 2013;15:569-75. 
[44] Pu KY, Shuhendler AJ, Jokerst JV, Mei JG, Gambhir SS, Bao ZN, et al. Semiconducting polymer nanoparticles as photoacoustic molecular imaging probes in living mice. Nat Nanotechnol. 2014;9:233-9. 

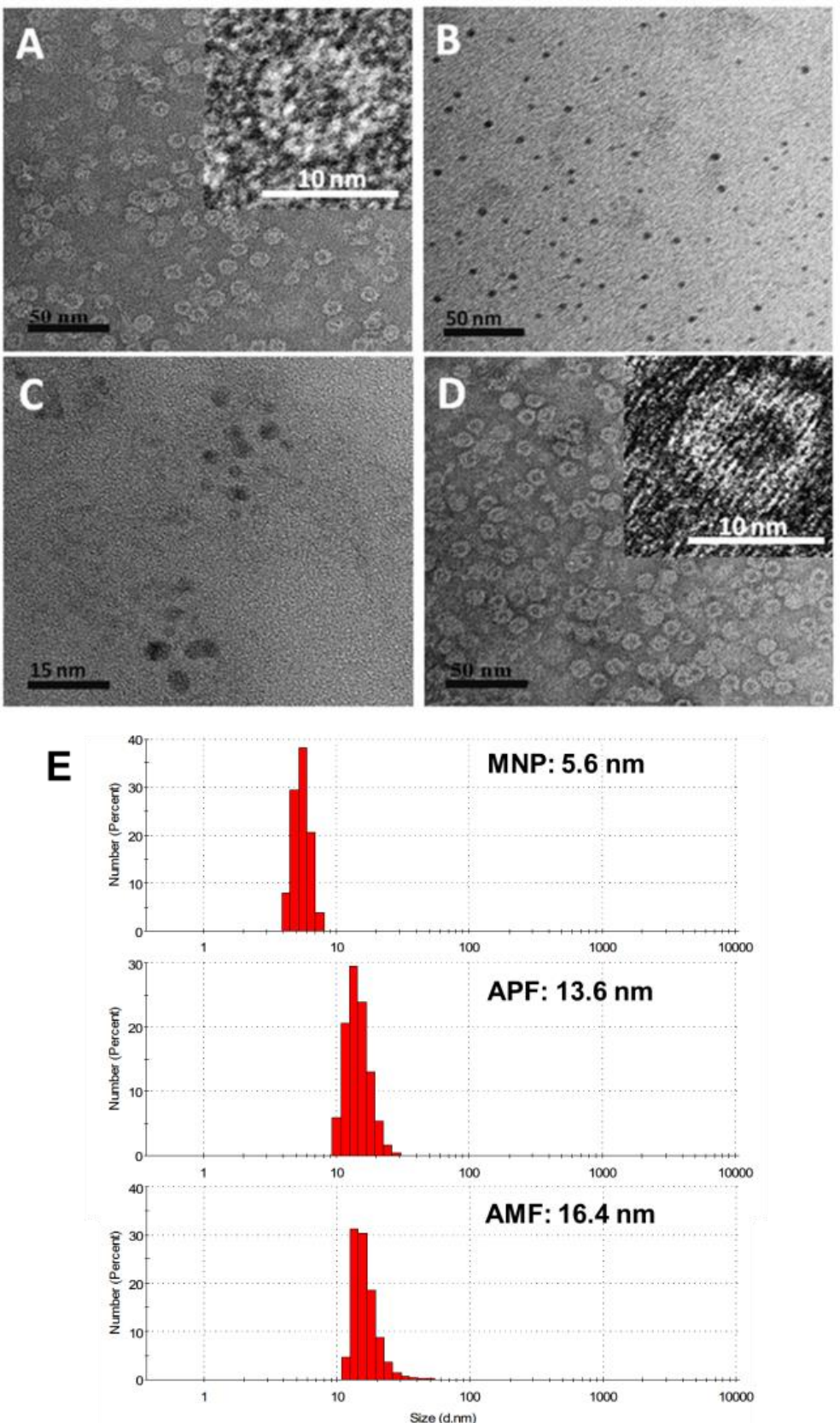

Fig. 2. AMF nanocages characterized in A (negative stained TEM images of APF) and D (negative stained images of AMF). B: Unstained TEM images of MNP. C: Fe-MNP in AMF. E: Diameters of MNP, APF and AMF by DLS. 


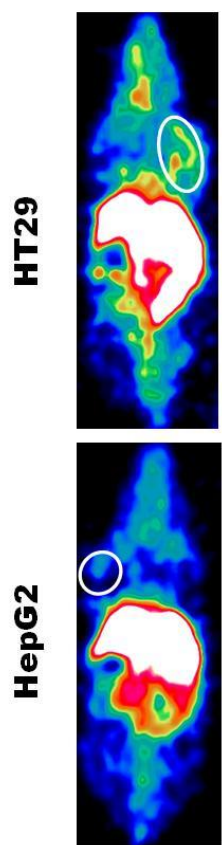

1h
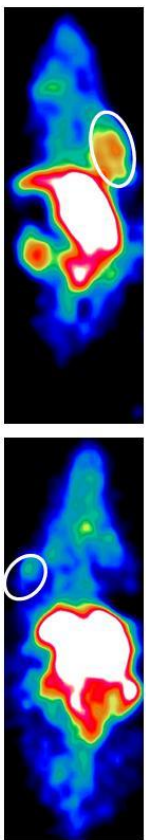

$2 h$
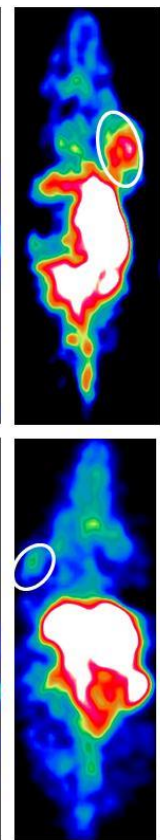

4h
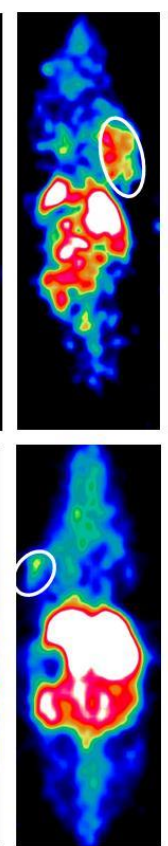

$18 h$
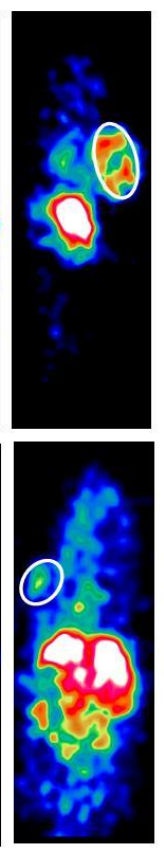

26h
$10 \mathrm{ID} \% / \mathrm{g}$

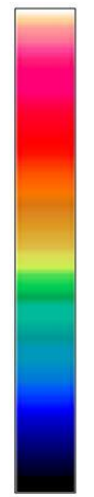

$0 \mathrm{ID} \% \mathrm{~g}$

Fig. 3. Representative coronal small animal PET images of HT29 (top) and HepG2 (bottom) tumor-bearing mice $(\mathrm{n}=4)$ at $1,2,4,18$ and $26 \mathrm{~h}$ after administration of ${ }^{64} \mathrm{Cu}$-AMF. White circles indicate tumors location.
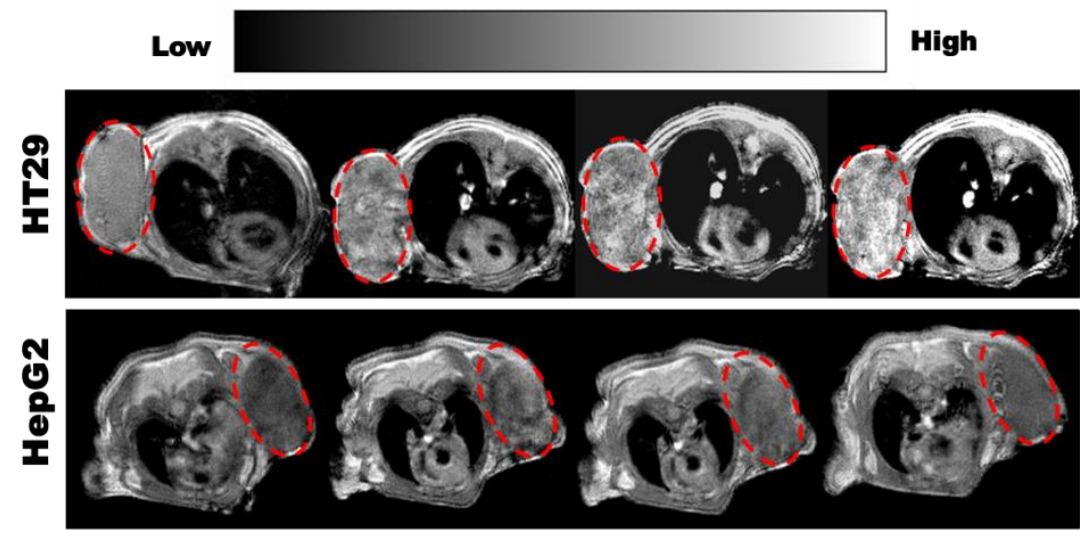

Pre

1h

2h

4h

Fig. 4. T1 MR images of HT29 (upper) and HepG2 (lower) tumor model after administration of $\mathrm{AMF}$, red circles indicate the area of tumors. 

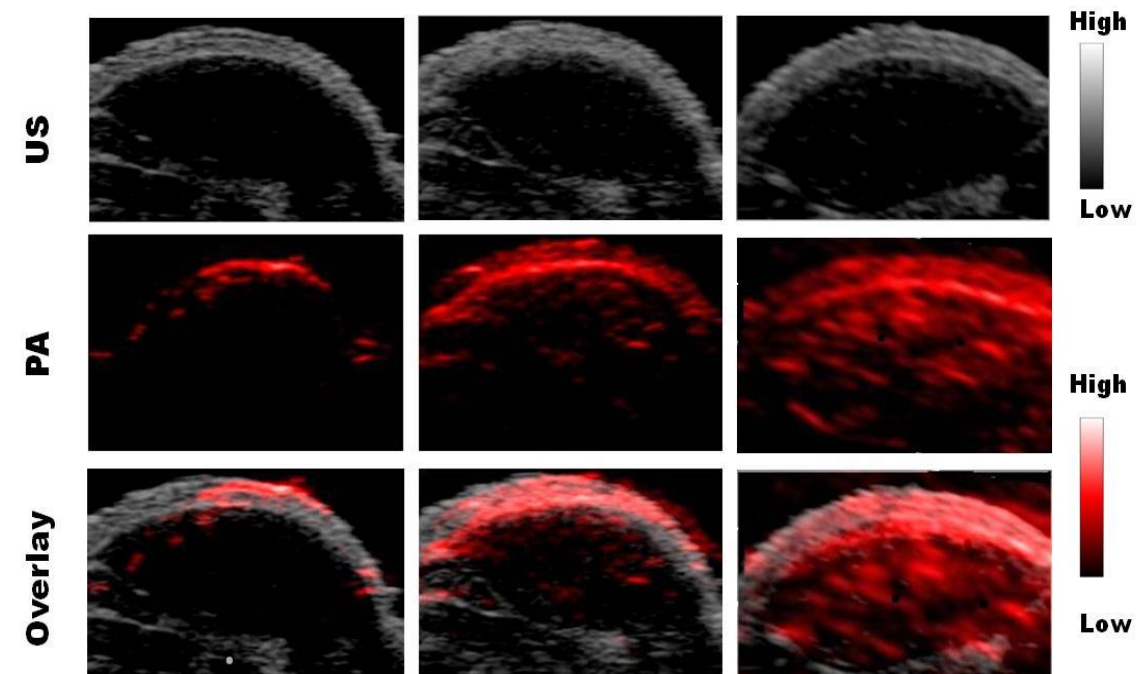

Pre

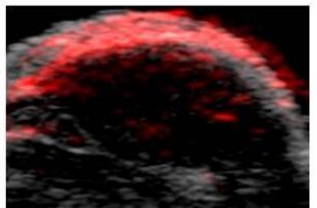

2h

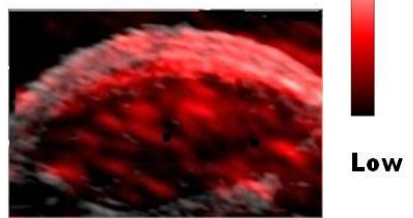

$4 h$

Fig. 5. The US (grey, top), PA (red, middle) and overlayed coronal sections (bottom) of HT29 tumor models before and after tail-vein injection of AMF nanocages. 